\title{
A Preparedness Model for Mother-Baby Linked Longitudinal Surveillance for Emerging Threats
}

\author{
Kate R. Woodworth ${ }^{1}$ (D) Megan R. Reynolds ${ }^{1} \cdot$ Veronica Burkel $^{1,2} \cdot$ Cymone Gates $^{3} \cdot$ Valorie Eckert $^{4}$. \\ Catherine McDermott ${ }^{5}$. Jerusha Barton ${ }^{6}$. Amanda Wilburn ${ }^{7}$. Umme-Aiman Halai ${ }^{8}$. Catherine M. Brown ${ }^{9}$. \\ Angelica Bocour ${ }^{10} \cdot$ Nicole Longcore $^{11} \cdot$ Lauren Orkis $^{12}$. Camille Delgado Lopez ${ }^{13}$. Lindsey Sizemore ${ }^{14}$. \\ Esther M. Ellis ${ }^{15}$. Sarah Schillie ${ }^{16} \cdot$ Neil Gupta $^{16}$. Virginia B. Bowen ${ }^{17}$. Elizabeth Torrone ${ }^{17}$. Sascha R. Ellington ${ }^{18}$. \\ Augustina Delaney ${ }^{1,2}$. Samantha M. Olson ${ }^{1,19} \cdot$ Nicole M. Roth $^{1,2} \cdot$ Florence Whitehill $^{1,20} \cdot$ Laura D. Zambrano $^{1}$. \\ Dana Meaney-Delman ${ }^{1} \cdot$ S. Nicole Fehrenbach ${ }^{1} \cdot$ Margaret A. Honein $^{1} \cdot$ Van T. Tong ${ }^{1} \cdot$ Suzanne M. Gilboa ${ }^{1}$
}

Accepted: 24 November 2020 / Published online: 4 January 2021

(c) Springer Science+Business Media, LLC, part of Springer Nature 2021

\begin{abstract}
Introduction Public health responses often lack the infrastructure to capture the impact of public health emergencies on pregnant women and infants, with limited mechanisms for linking pregnant women with their infants nationally to monitor long-term effects. In 2019, the Centers for Disease Control and Prevention (CDC), in close collaboration with state, local, and territorial health departments, began a 5-year initiative to establish population-based mother-baby linked longitudinal surveillance, the Surveillance for Emerging Threats to Mothers and Babies Network (SET-NET).

Objectives The objective of this report is to describe an expanded surveillance approach that leverages and modernizes existing surveillance systems to address the impact of emerging health threats during pregnancy on pregnant women and their infants.

Methods Mother-baby pairs are identified through prospective identification during pregnancy and/or identification of an infant with retrospective linking to maternal information. All data are obtained from existing data sources (e.g., electronic medical records, vital statistics, laboratory reports, and health department investigations and case reporting).

Results Variables were selected for inclusion to address key surveillance questions proposed by CDC and health department subject matter experts. General variables include maternal demographics and health history, pregnancy and infant outcomes, maternal and infant laboratory results, and child health outcomes up to the second birthday. Exposure-specific modular variables are included for hepatitis C, syphilis, and Coronavirus Disease 2019 (COVID-19). The system is structured into four relational datasets (maternal, pregnancy outcomes and birth, infant/child follow-up, and laboratory testing).

Discussion SET-NET provides a population-based mother-baby linked longitudinal surveillance approach and has already demonstrated rapid adaptation to COVID-19. This innovative approach leverages existing data sources and rapidly collects data and informs clinical guidance and practice. These data can help to reduce exposure risk and adverse outcomes among pregnant women and their infants, direct public health action, and strengthen public health systems.
\end{abstract}

Keywords Pregnancy $\cdot$ Congenital infection $\cdot$ Perinatal hepatitis C $\cdot$ Congenital syphilis $\cdot$ SARS-CoV-2 $\cdot$ COVID-19 . Surveillance

Kate R. Woodworth

vnt0@cdc.gov

Extended author information available on the last page of the article

\section{Significance Statement}

This report describes the methods of the Surveillance for Emerging Threats to Mothers and Babies Network (SETNET), a novel and flexible approach of linked-longitudinal mother-baby surveillance. By leveraging existing data sources and linking mothers and their infants, SET-NET can rapidly assess the impact of existing, re-emerging, and 
novel infectious disease threats to mothers and babies, to inform clinical guidance and practice, and direct public health action.

\section{Introduction}

Recent public health emergencies highlight the need for innovative and coordinated population-based pregnancy and infant surveillance (Frey et al. 2019). Pregnant women might be more susceptible to emerging infections or more severe illness compared with the general population and experience adverse pregnancy or birth outcomes not routinely captured through emergency surveillance efforts (Rasmussen and Hayes 2005). Public health responses have typically lacked rapid, nationally representative surveillance models to capture data and link pregnant women with their infants to monitor the effects of pregnancy exposures on outcomes over time (Frey et al. 2019).

Historically, surveillance approaches have been siloed by exposure with disparate definitions and collection methods. Existing surveillance systems have not been flexible enough to capture relevant data from the wide array of sources and time points needed to fully assess pregnancy and infant outcomes. These limitations pose a barrier to using data for public health and clinical decision-making. The U.S. Zika Pregnancy and Infant Registry (USZPIR) was established shortly after the initiation of the Centers for Disease Control and Prevention (CDC) Zika Virus Response and allowed public health officials to rapidly monitor and assess pregnancy and early childhood outcomes for $>7400$ U.S. pregnancies with laboratory evidence of possible Zika virus infection. By establishing a mother-baby linked longitudinal surveillance platform, rapidly collecting and analyzing data and translating and dissemination the information, this national registry was a key source of up-to-date information to inform clinicians, public health officials, women and families, and the public (Honein et al. 2017). Adapting and improving upon this approach, in 2019, CDC, in collaboration with state, local, and territorial health departments, began a 5-year initiative to establish population-based mother-baby linked longitudinal surveillance for other exposures. This adapted surveillance approach is known as the Surveillance for Emerging Threats to Mothers and Babies Network (SET-NET, https://www.cdc.gov/ncbddd/aboutus/ pregnancy/emerging-threats.html).

In year one of funding, SET-NET expanded to hepatitis $\mathrm{C}$ and syphilis. At the beginning of the Coronavirus Disease 2019 (COVID-19) pandemic, SET-NET was rapidly adapted to capture information about severe acute respiratory syndrome coronavirus (SARS-CoV-2), the virus that causes COVID-19. This report provides methodologic details about the development and implementation of SET-NET.

\section{Methods}

\section{Participating Jurisdictions}

CDC established SET-NET in collaboration with the health departments of Arizona, California (except Los Angeles County), Los Angeles County, Florida, Georgia, Kentucky, Massachusetts, New York state (except New York City), New York City, Pennsylvania, Puerto Rico, Tennessee, and the U.S. Virgin Islands in year one (Fig. 1). Jurisdictions receive funding through the Epidemiology and Laboratory Capacity for Prevention and Control of Emerging Infectious Diseases (ELC) Cooperative Agreement (ELC CK19-1904) or through contractual mechanisms.

\section{Purpose}

The main objective of SET-NET is to leverage existing surveillance systems and data sources to assess the impact of emerging health threats on pregnant women and their infants/children.

\section{Case Ascertainment}

Methods of case ascertainment vary by exposure and jurisdiction and include prospective identification of a pregnant woman with the exposure of interest and/or identification of the infant with retrospective linking to maternal information. Current exposures of interest within SET-NET are reportable infectious diseases, so ascertaining pregnancy status may depend on reporting at the time of case confirmation. Jurisdictions use linkages between available datasets to identify pregnant/postpartum women (e.g., matching between women of reproductive age in hepatitis $\mathrm{C}$ laboratory reporting with prenatal screening or vital statistics) as well as to identify infants (e.g., immunization registries to identify most recent primary care provider).

\section{Data Sources}

Data elements are obtained from existing data sources with no need for additional primary data collection (e.g., patient interviews, clinical visits, or laboratory reports). Examples of data sources used by participating jurisdictions include

- Existing state data systems (e.g., vital statistics, electronic laboratory reporting, and birth defects surveillance) 


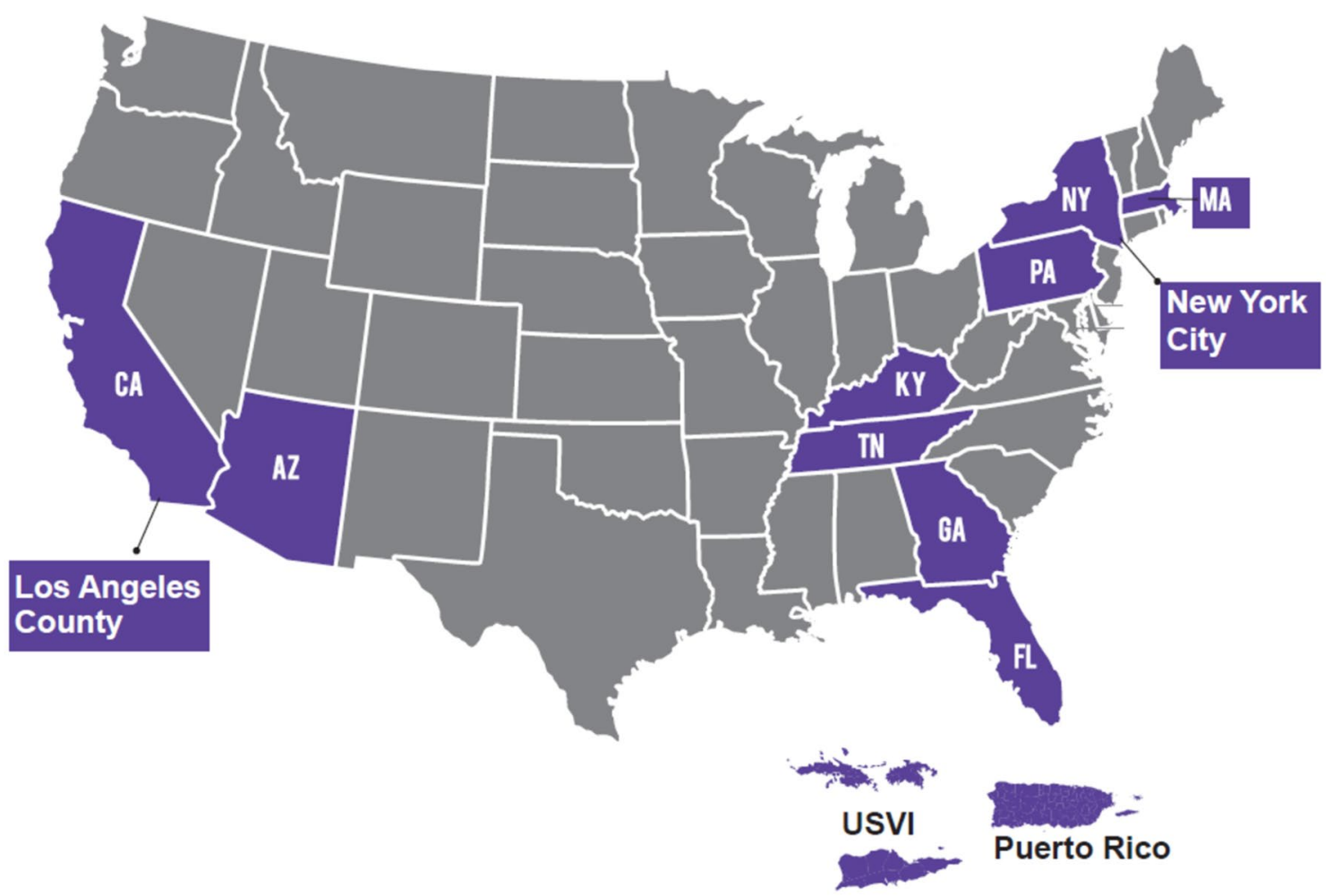

Fig. 1 Jurisdictions funded to participate in the Surveillance for Emerging Threats to Mothers and Babies Network (SET-NET) in year one

- Records from routine case investigations and reports (e.g., case interviews, disease investigations, and case report forms)

- Medical records from hospitals and healthcare providers' offices (e.g., prenatal records, maternal hospitalization records, and infant follow-up medical records)

Electronically linked data sources are encouraged when possible in order to decrease burden of manual chart abstraction and data entry and prevent duplication of effort.

\section{General and Modular Variables}

Since 2018, the National Center on Birth Defects and Developmental Disabilities has led a cross-agency effort to develop standardized data elements for conducting mother-baby linked longitudinal surveillance. The proposed data elements serve as the foundation for SET-NET's "general" variables, which pertain to all mother-baby pairs, regardless of the exposure of interest (Fig. 2). Exposurespecific or "modular" variables complement the general variables by providing information for mother-baby pairs about the exposure of interest (Fig. 3). Modular variables were selected to align with a variety of data sources and variables found in reviews of the literature and were reviewed by a team of experts in obstetrics, pediatrics, epidemiology, and informatics with a focus on key questions as well as potential for data capture. Together, general and modular variables are selected to align with key surveillance questions for each exposure, while striving to minimize burden and ensure quality data.

CDC has an Assurance of Confidentiality (https:// www.cdc.gov/od/science/integrity/confidentiality/) for data reported through SET-NET, consistent with Section 308(d) of the Public Health Service Act (42 U.S.C. Section $242 \mathrm{~m}(\mathrm{~d})$ ), which provides the highest level of protection for identifiable and potentially identifiable data collected by health departments and reported to CDC. The legal authority for public health and disease control varies by jurisdiction and may affect surveillance approaches, access to data sources, and case ascertainment. In the following section, we present key questions, variables, and follow-up for each exposure.

\section{Results}

\section{Hepatitis C Module}

Hepatitis $\mathrm{C}$ virus (HCV) causes acute and chronic infection that can lead to long-term morbidity and mortality. Pregnant women with $\mathrm{HCV}$ infection can pass the virus to their 


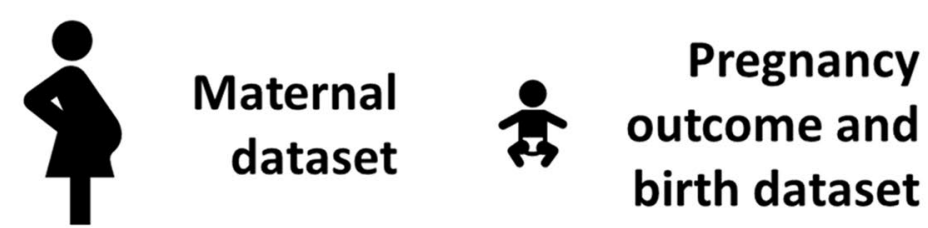

Infant

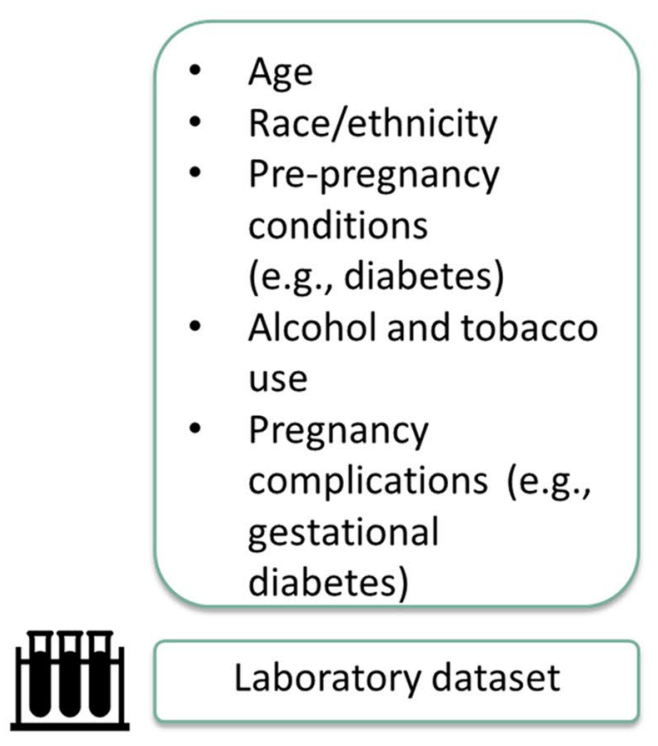

- Pregnancy outcome

- Gestational age

- Infant sex

- Anthropometry (e.g., height, weight, head circumference)

- Physical exam

- Birth defects

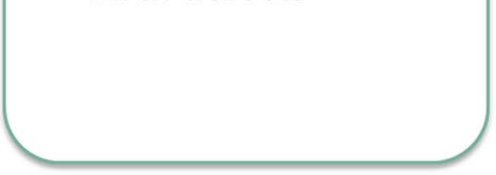

Laboratory dataset follow-up

dataset

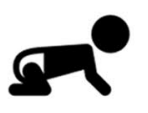

Fig. 2 General variables included in the Surveillance for Emerging Threats to Mothers and Babies Network (SET-NET)

Fig. 3 Modular data structure for Surveillance for Emerging Threats to Mothers and Babies Network (SET-NET), including general variables to be collected for all mother-baby pairs, and modular variables to be collected based on exposure of interest (e.g., general variables and variables included in the hepatitis $\mathrm{C}$ module are included for hepatitis $\mathrm{C}$ surveillance)
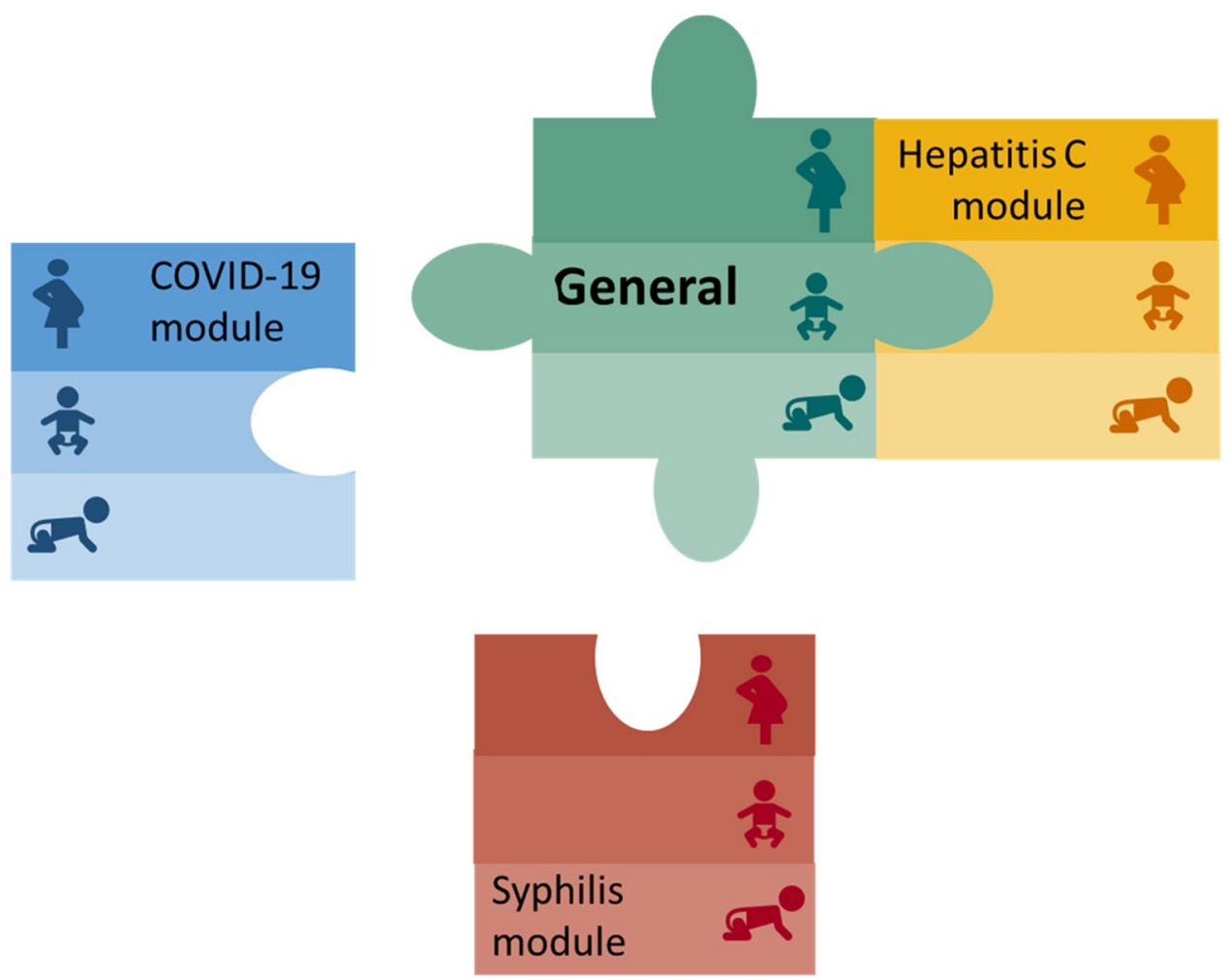
infants, occurring in about $6 \%$ of $\mathrm{HCV}$-exposed pregnancies (Benova et al. 2014). The incidence of HCV infection in the United States has been increasing since 2009 among young adults, including women of reproductive age and pregnant women, concurrent with the opioid crisis (CDC 2019; Patrick et al. 2017). Surveillance for perinatal hepatitis $\mathrm{C}$ is incomplete and likely underestimates the burden of $\mathrm{HCV}$ in pregnancy, due to limited surveillance resources, lack of identification of infected pregnant women, and inadequate tracking and testing of exposed infants (Chappell et al. 2018). CDC recently released recommendations for universal hepatitis $\mathrm{C}$ screening of all adults and pregnant women during each pregnancy (Schillie et al. 2020). With these updated recommendations and newly available curative treatments for children $\geq 3$ years old and for pregnant women in clinical trials, the landscape of HCV infection surveillance, prevention, and treatment is rapidly changing. SET-NET aims to describe the timing of hepatitis $\mathrm{C}$ diagnosis (pre-pregnancy or during pregnancy), referral for treatment postpartum, and adverse fetal and birth outcomes among HCV-exposed pregnancies. Additionally, SET-NET aims to characterize loss to follow-up, testing, and clinical complications among HCV-exposed infants by maternal and birth characteristics, with specific inclusion criteria for the population of interest for each exposure (Table 1).

Exposure-specific variables for $\mathrm{HCV}$ infection include maternal risk factors (e.g., incarceration and injection drug use), diagnosis and treatment characteristics, delivery characteristics (e.g., use of internal fetal monitoring and prolonged rupture of membranes), infant testing information, and infant clinical complications. Follow-up for children includes 12- and 24-month well child visits, since recommended testing for RNA or serology is expected by these time periods [https://wwwn.cdc.gov/nndss/conditions/hepat itis-c-perinatal-infection/case-definition/2018/].

Given the large case burden of infants with in utero exposure to $\mathrm{HCV}$, a sampling scheme was developed to prioritize medical record abstraction of infant follow-up data. Medical records are abstracted for all children with positive $\mathrm{HCV}$ testing and for a random selection of children with

Table 1 Inclusion criteria for hepatitis C virus (HCV), syphilis, and 2019 coronavirus disease (COVID-19) in the Surveillance for Emerging Threats to Mothers and Babies Network (SET-NET)

\begin{tabular}{|c|c|c|c|}
\hline & Hepatitis C & Syphilis & COVID-19 \\
\hline $\begin{array}{l}\text { Inclusion based on maternal } \\
\text { testing }\end{array}$ & $\begin{array}{l}\text { (1) Pregnant women who are } \\
\text { HCV RNA+ during pregnancy, } \\
\text { or prior to pregnancy without } \\
\text { evidence of treatment or clear- } \\
\text { ance and; } \\
\text { (2) The infants or chil- } \\
\text { dren }<3 \text { years of age born of } \\
\text { that pregnancy, regardless of } \\
\text { laboratory evidence of hepatitis } \\
\text { C infection in the child }\end{array}$ & $\begin{array}{l}\text { (1) Pregnant women who meet } \\
\text { Council of State and Territorial } \\
\text { Epidemiologists (CSTE) case } \\
\text { definition }{ }^{\mathrm{a}} \text { for syphilis at any } \\
\text { point during pregnancy and; } \\
\text { (2) The infants or chil- } \\
\text { dren }<3 \text { years of age born of } \\
\text { that pregnancy, regardless of } \\
\text { laboratory or clinical evidence } \\
\text { of congenital syphilis in the } \\
\text { child }\end{array}$ & $\begin{array}{l}\text { (1) Pregnant women who meet the } \\
\text { CSTE laboratory criteria for con- } \\
\text { firmed SARS-CoV-2 infection }{ }^{c} \\
\text { at any point during pregnancy } \\
\text { and; } \\
\text { (2) The neonates born of that preg- } \\
\text { nancy, regardless of laboratory } \\
\text { evidence of SARS-CoV-2 in the } \\
\text { neonate }\end{array}$ \\
\hline $\begin{array}{l}\text { Inclusion based on infant/child's } \\
\text { testing }\end{array}$ & $\begin{array}{l}\text { (1) Infants or children }<3 \text { years } \\
\text { of age who meet the CSTE case } \\
\text { definition for perinatal hepatitis } \\
\text { C, and; } \\
\text { (2)Their birth mothers, regard- } \\
\text { less of laboratory evidence of } \\
\text { maternal HCV infection }\end{array}$ & $\begin{array}{l}\text { (1) Stillbirths, infants or chil- } \\
\text { dren }<3 \text { years of age who meet } \\
\text { the probable or confirmed CSTE } \\
\text { case definitions for congenital } \\
\text { syphilis or syphilitic stillbirth } \\
\text { and; } \\
\text { (2) Their birth mothers regard- } \\
\text { less of laboratory evidence of } \\
\text { maternal syphilis infection }\end{array}$ & Not applicable \\
\hline $\begin{array}{l}\text { Pregnancy completion or birth } \\
\text { date }\end{array}$ & On or after January 1, 2018 & On or after January 1, 2018 & On or after January 1, 2020 \\
\hline
\end{tabular}

All mother-baby pairs included must reside within a participating jurisdiction

${ }^{a}$ This may include persons with primary, secondary, early non-primary non-secondary syphilis and unknown duration or late syphilitic infections. https://wwwn.cdc.gov/nndss/conditions/syphilis/case-definition/2018/

${ }^{\mathrm{b}}$ The CSTE case definition for congenital syphilis includes stillbirths and infants born to mothers with untreated or inadequately treated syphilis and infants with laboratory evidence of congenital syphilis (direct detection of Treponema pallidum or a reactive nontreponemal syphilis test) as well as clinical or radiographic findings or cerebral spinal fluid analysis consistent with congenital syphilis. https://wwwn.cdc.gov/nndss/condi tions/syphilis/case-definition/2018/

${ }^{\mathrm{c}}$ The CSTE interim case definition for laboratory confirmed SARS-CoV-2 infection includes persons with detection of SARS-CoV-2 ribonucleic acid (RNA) in a clinical specimen using a molecular amplification detection test. https://wwwn.cdc.gov/nndss/conditions/coronavirus-disease2019-covid-19/case-definition/2020/ 
negative or inappropriate testing (e.g., antibody testing before 18 months of age), or without testing results available as some jurisdictions do not receive negative testing results reliably.

\section{Syphilis Module}

A pregnant woman with syphilis can face serious health problems if the infection is not treated, and can pass the infection to her fetus in utero, resulting in congenital syphilis. Congenital syphilis can result in miscarriage, stillbirth, neonatal death, and severe lifelong physical problems to the infant (Gomez et al. 2013). CDC recommends that all pregnant women be screened for syphilis at the first prenatal visit and individuals at high risk of acquiring syphilis later in pregnancy be re-tested at 28 weeks gestation and again at delivery (Workowski et al. 2015). Syphilis during pregnancy can be cured with antibiotics, but, if left untreated, approximately $66 \%$ of pregnant women with syphilis experience an adverse pregnancy outcome (Gomez et al. 2013). Increasing trends in syphilitic infections among women of reproductive age and increases in congenital syphilis cases are worrisome (Bowen et al. 2015). The availability of an effective treatment alone is not enough to stem this trend, and there are multiple missed opportunities for diagnosis and treatment (Kimball et al. 2020).

Syphilis, both congenital infections and infections among adults, is a nationally notifiable condition, with health departments sending case notifications to CDC through the National Notifiable Diseases Surveillance System (NNDSS); however, existing surveillance at the federal level is limited by the inability to link case notifications for pregnant women with syphilis to case notifications for infants meeting the congenital syphilis case definition (https://wwwn. cdc.gov/nndss/conditions/congenital-syphilis/case-definition /2018/). Therefore, the proportion of maternal infections that result in congenital transmission cannot be determined at the national level. Furthermore, information on infants prenatally exposed to syphilis who do not meet the case definition for congenital syphilis are not currently tracked nationally but could help further inform prevention efforts. Finally, NNDSS captures information on congenital syphilis cases at time of diagnosis (usually at birth) and does not include longitudinal outcomes (e.g., neurodevelopmental outcomes). Information about the long-term consequences of congenital syphilis is critical to more fully describe the burden of this preventable infection. SET-NET aims to describe the diagnosis and treatment of pregnant women, the frequency of adverse pregnancy outcomes and Council of State and Territorial Epidemiologists (CSTE) case statuses among infants by maternal sociodemographic and clinical characteristics, the frequency and range of infant and early childhood clinical and developmental outcomes by infant case status, and characterize loss to follow-up by various sociodemographic and clinical characteristics.

Exposure-specific variables for syphilis are aligned as much as possible with NNDSS message mapping guides for sending syphilis case notifications to CDC (https://wwwn. cdc.gov/nndss/case-notification/message-mapping-guide s.html) and include maternal clinical (e.g., HIV status) and behavioral (e.g., substance use) characteristics, diagnosis and treatment information, clinical signs of congenital syphilis, infant testing results and case status, and infant imaging results and clinical complications. Time points for infant follow-up medical record abstraction include the 2-, 6-, 12-, 18-, and 24-month well child visits.

\section{COVID-19 Module}

In late 2019, the novel coronavirus SARS-CoV-2 which causes COVID-19 emerged, rapidly evolving into a global pandemic of historic proportions, causing many pregnant women, their families, and their healthcare providers to want to better understand the impact of this novel virus on pregnancy. Pregnant women might be at an increased risk for severe disease compared with non-pregnant women of reproductive age, and much remains unknown about the effect of SARS-CoV-2 infection on pregnancy and infant outcomes (Ellington et al. 2020). Traditional infectious disease surveillance methods capture point-in-time outcomes, typically around time of infection, and national case reporting does not capture data beyond a binary pregnancy status to comprehensively describe pregnancy outcomes. Mother-baby linked longitudinal surveillance is necessary to understand the full impact of COVID-19 in pregnancy on the health of pregnant women and their infants. Fortunately, the SETNET framework was poised for rapid expansion for COVID19. SET-NET aims to describe risk factors for disease, clinical course, complications, and treatment among pregnant women with COVID-19; the frequency and range of pregnancy and birth outcomes and characterization by maternal demographic and clinical characteristics; the frequency, timing, and characteristics of neonatal testing and infection; and description of rooming-in (whether infant stayed in mother's room) and in-hospital breastfeeding practices (https://www. cdc.gov/coronavirus/2019-ncov/cases-updates/special-popul ations/pregnancy-data-on-covid-19.html).

SET-NET COVID-19 surveillance is restricted to women with laboratory confirmed infection. As opposed to other exposures of interest, neonates who test positive for SARSCoV-2 but whose mother tested negative or whose testing status is unknown will not be included due to challenges in determining post-natal horizontal transmission vs. vertical transmission. Additionally, while there have been limited reports of perinatal SARS-CoV-2 infection, currently these 
routes of transmission seem to occur rarely, and the clinical significance is unknown (Kirtsman et al. 2020).

Data elements include maternal symptoms, complications, and treatment; delivery mode and induction; infant symptoms, complications, test results, and treatment; and whether the infant roomed-in, was ever fed maternal breastmilk (direct or expressed). Long-term consequences of SARS-CoV-2 infection are unknown. Timepoints for follow up include the first newborn visit, and the 2- and 6-month well child visits. Similar to HCV, a population-based sampling scheme has been developed for jurisdictions with high burden.

\section{Data Structure}

For all exposures, data on each mother-baby pair are transmitted to CDC as four relational datasets: maternal, pregnancy outcome and birth, infant follow-up, and laboratory testing (Fig. 2). This structure was selected based on best practices and experiences from prior data collection projects for mother-baby pairs. The relational dataset structure allows for multiple infants from one pregnancy and multiple longitudinal follow-up visits for each infant linked by a pregnancy identification number (ID). The maternal dataset includes information regarding the pregnant woman's demographics, health history, prenatal care, and pregnancy complications. The pregnancy outcome and birth dataset capture information on birth outcomes, including pregnancy loss, stillbirth, and live birth, as well as care and complications during birth hospitalization, for each fetus. Data for multiple gestation pregnancies are linked by pregnancy ID. The infant/child follow-up dataset longitudinally captures information after birth hospitalization. Follow-up data are collected by infant and timepoint, which are flexible and vary for the exposure of interest (e.g., 12-months and 24-months for hepatitis C). Finally, the laboratory testing dataset includes laboratory results pertaining to either the woman or the infant(s) and allows for an infinite number of tests. Laboratory data are stored based on specimen, test type, and date for the pregnant woman or the infant.

\section{Discussion}

SET-NET provides an approach to population-based mother-baby linked longitudinal surveillance that CDC and health departments can rapidly adapt to conduct surveillance for emerging threats. This innovative and relational surveillance approach leverages existing data sources and will inform clinical guidance and practice patterns to reduce maternal, fetal, and infant risk of exposure to SARS-CoV-2 and adverse outcomes, direct public health action, and strengthen public health systems. The system has already proven to be flexible and readily adaptable to the new threat of COVID-19, with completion of a data dictionary by May 2020 , receipt of data relevant to pregnancies affected by COVID- 19 by July 2020, and preliminary public reporting anticipated in fall 2020. Additional funding in future years would allow for further expansion to additional jurisdictions.

Strengths of this approach include improved efficiency through linkages of existing data systems, such as vital statistics and electronic laboratory reporting, and the development of a flexible network that can adapt to additional emerging threats. The approach sets standards for consistent and systematic surveillance for mother-baby pairs and is adaptable across exposures. Compared with smaller research studies, surveillance data can capture population-based and jurisdictional-level exposure and outcome information to assess prevalence of rare outcomes and assist jurisdictions with preparedness and planning efforts. Additionally, it has the potential to provide more timely data in order to more effectively inform public health action. However, one limitation of this approach is that surveillance data lack a comparison group. Any associations identified will require additional research to distinguish between association and causality.

Challenges observed during the first year of SET-NET surveillance include variations in jurisdictions' ability to collect all variables, including regulatory restrictions and capacity. While SET-NET aims to improve efficiencies in surveillance, medical record abstraction is necessary for some variables. Challenges to identification of mother-baby pairs include under reporting of pregnancy status on provider or electronic laboratory reports, late or no prenatal care, and custody or name changes after birth hospitalization. Jurisdictions continue to explore and share best practices to improve case ascertainment (e.g., identification of pregnancy status by linking with vital records) and reduce loss to follow-up (e.g., linkages with immunization registries) (Wingate et al. 2019; Alaali et al. 2020).

Collaboration between exposure-specific surveillance groups and subject matter experts within sections of health departments responsible for maternal and child health, at the federal, state and local level, is necessary for successful surveillance and translation into public health action and practical clinical guidance. These groups are often in separate divisions within a health department (e.g., infectious disease and birth defects surveillance) and may be mutually unaware of the range of resources available to support linked longitudinal surveillance efforts. Additionally, there may be regulations that prevent sharing of data across divisions. Collaboration, communication, and buy in from all key stakeholders ensures efficient use of resources, reduces duplicate efforts, and improves communication of findings.

Despite disparate historical surveillance methods and underlying pathology of exposures of interest, the SETNET approach for surveillance of hepatitis $\mathrm{C}$ and syphilis 
in pregnancy has already demonstrated the utility of this model for existing, reemerging infectious diseases and the adaptation for COVID-19 has demonstrated the flexibility and utility for new emerging threats. Use of timely and actionable surveillance data gathered through SET-NET can help protect and improve the lives of pregnant women and their infants in response to a multitude of current and future emerging threats.

Acknowledgements Amanda Akosa, Eagle Global Scientific, CDC; Elizabeth Lewis, Chickasaw Health Consulting, CDC; John Nahabedian, Eagle Global Scientific, CDC; Breanne Anderson, Arizona Department of Health Services; Rebecca Scranton, Arizona Department of Health Services; Andrea Morrison, Florida Department of Health; Amanda Elmore, Florida Department of Health; Heather Lake-Burger, Florida Department of Health; Danielle Stanek, Florida Department of Health; Michael Bryan, Georgia Department of Public Health; Teri' Willabus, Georgia Department of Public Health; Cynthia Carpentieri, Georgia Department of Public Health; Caleb Lyu, Los Angeles County Department of Public Health; Clara Chang, Los Angeles County Department of Public Health; Craig Vincent-Jones, Los Angeles County Department of Public Health; Emily Barnes, Los Angeles County Department of Public Health; Mireille Ibrahim, Los Angeles County Department of Public Health; Meredith Haddix, Los Angeles County Department of Public Health; Prabhu Gounder, Los Angeles County Department of Public Health; Van Ngo, Los Angeles County Department of Public Health; Anthony Osinski, Massachusetts Department of Public Health; Mahsa Yazdy, Massachusetts Department of Public Health; Eirini Nestoridi, Massachusetts Department of Public Health; Elizabeth Dufort, New York State Department of Health; Zahra Alaali, New York State Department of Health; Pauline Santos, New York State Department of Health; Nadia Thomas, New York State Department of Health; Ellen Lee, New York City Department of Health \& Mental Hygiene; Ann Winters, New York City Department of Health \& Mental Hygiene; Miguel Valencia Prado, Puerto Rico Department of Health; Elizabeth Harvey, Tennessee Department of Health; Heather Wingate, Tennessee Department of Health; Laura Price, Tennessee Department of Health; and Katherine Lolley, Tennessee Department of Health.

Funding This study was performed as regular work of the Centers for Disease Control and Prevention. This work is supported by the Epidemiology and Laboratory Capacity for Prevention and Control of Emerging Infectious Diseases (ELC) Cooperative Agreement (ELC CK19-1904).

\section{Compliance with Ethical Standards}

Conflict of interest The authors of this manuscript have no conflicts of interest to disclose. The findings and conclusions in this report are those of the authors and do not necessarily represent the official position of the Centers for Disease Control and Prevention.

\section{References}

Alaali, Z. S., Longcore, N. D., Santos, P., Glaze, V. H., \& Ahmad, N. (2020). Methods of tracking newborns: New York State Zika Pregnancy and Infant Registry, 2015-2017. American Journal of Public Health, 110(2), 216-221. https://doi.org/10.2105/ AJPH.2019.305406.
Benova, L., Mohamoud, Y. A., Calvert, C., \& Abu-Raddad, L. J. (2014). Vertical transmission of hepatitis C virus: systematic review and meta-analysis. Clinical Infectious Diseases, 59(6), 765-773. https://doi.org/10.1093/cid/ciu447.

Bowen, V., Su, J., Torrone, E., Kidd, S., \& Weinstock, H. (2015). Increase in incidence of congenital syphilis-United States, 2012-2014. Morbidity and Mortality Weekly Report, 64(44), 1241-1245. https://doi.org/10.15585/mmwr.mm6444a3.

Centers for Disease Control and Prevention. (2019). Surveillance for viral hepatitis-United States, 2017. Retrieved February 7, 2020 from https://www.cdc.gov/hepatitis/statistics/2017survei llance/index.htm.

Chappell, C. A., Hillier, S. L., Crowe, D., Meyn, L. A., Bogen, D. L., \& Krans, E. E. (2018). Hepatitis C virus screening among children exposed during pregnancy. Pediatrics, 141(6), e20173273. https://doi.org/10.1542/peds.2017-3273.

Council of State and Territorial Epidemiologists. (2015). Congenital syphilis (Treponema pallidum) 2015 case definition. Retrieved May 19, 2020 from https://wwwn.cdc.gov/nndss/conditions/ congenital-syphilis/case-definition/2015/.

Council of State and Territorial Epidemiologists. (2018). Hepatitis C, perinatal infection. Retrieved May 19, 2020 from https:// wwwn.cdc.gov/nndss/conditions/hepatitis-c-perinatal-infection/ case-definition/2018/.

Ellington, S., Strid, P., Tong, V. T., Woodworth, K., Galang, R. R., Zambrano, L. D., et al. (2020). Characteristics of women of reproductive age with laboratory-confirmed SARS-CoV-2 infection by pregnancy status-United States, January 22-June 7, 2020. Morbidity and Mortality Weekly Report, 69(25), 769-775. https://doi.org/10.15585/mmwr.mm6925a1.

Frey, M. T., Meaney-Delman, D., Bowen, V., Yazdy, M. M., Watkins, S. M., Thorpe, P. G., et al. (2019). Surveillance for emerging threats to pregnant women and infants. Journal of Women's Health (Larchmt), 28(8), 1031-1036. https://doi.org/10.1089/ jwh.2019.7943.

Gomez, G. B., Kamb, M. L., Newman, L. M., Mark, J., Broutet, N., \& Hawkes, S. J. (2013). Untreated maternal syphilis and adverse outcomes of pregnancy: A systematic review and meta-analysis. Bulletin of the World Health Organization, 91(3), 217-226. https://doi.org/10.2471/BLT.12.107623.

Honein, M. A., Dawson, A. L., Petersen, E. E., Jones, A. M., Lee, E. H., Yazdy, M. M., et al. (2017). Birth defects among fetuses and infants of US women with evidence of possible Zika virus infection during pregnancy. Journal of the American Medical Association, 317(1), 59-68. https://doi.org/10.1001/jama.2016.19006.

Kimball, A., Torrone, E., Miele, K., Bachmann, L., Thorpe, P., Weinstock, H., et al. (2020). Missed opportunities for prevention of congenital syphilis-United States, 2018. Morbidity and Mortality Weekly Report, 69(22), 661-665. https://doi.org/10.15585 /mmwr.mm6922a1.

Kirtsman, M., Diambomba, Y., Poutanen, S. M., Malinowski, A. K., Vlachodimitropoulou, E. W., Parks, T., et al. (2020). Probable congenital SARS-CoV-2 infection in a neonate born to a woman with active SARS-CoV-2 infection. Canadian Medical Association Journal, 192(24), E647-E650. https://doi.org/10.1503/ cmaj. 200821.

Patrick, S. W., Bauer, A. M., Warren, M. D., Jones, T. F., \& Wester, C. (2017). Hepatitis $C$ virus infection among women giving birth-Tennessee and United States, 2009-2014. Morbidity and Mortality Weekly Report, 66(18), 470-473. https://doi. org/10.15585/mmwr.mm6618a3.

Rasmussen, S. A., \& Hayes, E. B. (2005). Public health approach to emerging infections among pregnant women. American Journal of Public Health, 95(11), 1942-1944. https://doi.org/10.2105/ AJPH.2004.054957. 
Schillie, S., Wester, C., Osborne, M., Wesolowski, L., \& Ryerson, A. B. (2020). CDC recommendations for hepatitis C screening among adults-United States, 2020. MMWR Recommendations and Reports, 69(2), 1-17. https://doi.org/10.15585/mmwr.rr6902a1.

Wingate, H., Sizemore, L., Black, J., \& Wester, C. (2019). Retrospective surveillance of perinatal hepatitis $\mathrm{C}$ virus exposure-Tennessee, 2013-2017 [Conference presentation abstract]. International Society for Disease Conference, San Diego, CA.

Workowski, K. A., Bolan, G. A., \& Centers for Disease Control and Prevention. (2015). Sexually transmitted diseases treatment guidelines, 2015. MMWR Recommendations and Reports, 64(RR03), 1-137.

Publisher's Note Springer Nature remains neutral with regard to jurisdictional claims in published maps and institutional affiliations.

\section{Affiliations}

Kate R. Woodworth ${ }^{1}$ (1) $\cdot$ Megan R. Reynolds ${ }^{1} \cdot$ Veronica Burkel $^{1,2} \cdot$ Cymone Gates $^{3} \cdot$ Valorie Eckert $^{4}$. Catherine McDermott ${ }^{5}$. Jerusha Barton ${ }^{6}$. Amanda Wilburn ${ }^{7}$. Umme-Aiman Halai ${ }^{8}$. Catherine M. Brown ${ }^{9}$. Angelica Bocour ${ }^{10} \cdot$ Nicole Longcore $^{11} \cdot$ Lauren Orkis $^{12}$. Camille Delgado Lopez ${ }^{13} \cdot$ Lindsey Sizemore $^{14}$. Esther M. Ellis ${ }^{15}$. Sarah Schillie ${ }^{16} \cdot$ Neil Gupta $^{16} \cdot$ Virginia B. Bowen $^{17}$. Elizabeth Torrone ${ }^{17}$. Sascha R. Ellington ${ }^{18}$. Augustina Delaney ${ }^{1,2}$. Samantha M. Olson ${ }^{1,19} \cdot$ Nicole M. Roth $^{1,2}$. Florence Whitehill, ${ }^{1,20}$. Laura D. Zambrano ${ }^{1}$. Dana Meaney-Delman ${ }^{1}$. S. Nicole Fehrenbach ${ }^{1} \cdot$ Margaret A. Honein $^{1} \cdot \operatorname{Van}$ T. Tong ${ }^{1} \cdot$ Suzanne M. Gilboa $^{1}$

1 Division of Birth Defects and Infant Disorders, National Center on Birth Defects and Developmental Disabilities, Centers for Disease Control and Prevention (CDC), 4770 Buford Highway NE, Atlanta, GA 30341, USA

2 Eagle Medical Services and Eagle Global Scientific, LLC, Atlanta, USA

3 Arizona Department of Health Services, Phoenix, USA

4 California Department of Public Health, Sacramento, USA

5 Florida Department of Health, Tallahassee, USA

6 Georgia Department of Public Health, Atlanta, USA

7 Kentucky Department for Public Health, Frankfort, USA

8 Los Angeles County Department of Public Health, Los Angeles, USA

9 Massachusetts Department of Public Health, Boston, USA

10 New York City Department of Health \& Mental Hygiene, Long Island City, USA

11 New York State Department of Health, Albany, USA
13 Puerto Rico Department of Health, San Juan, USA

14 Tennessee Department of Health, Nashville, USA

15 U.S. Virgin Islands Department of Health, Christiansted, USA

16 Division of Viral Hepatitis, National Center for HIV/AIDS, Viral Hepatitis, STD, and TB Prevention, CDC, Atlanta, USA

17 Division of STD Prevention, National Center for HIV, Hepatitis, STD, and TB Prevention, CDC, Atlanta, USA

18 Division of Reproductive Health, National Center for Chronic Disease Prevention and Health Promotion, CDC, Atlanta, USA

19 G2S Corporation, San Antonio, USA

20 Oak Ridge Institute for Science and Education, Oak Ridge, USA 